\title{
Errorless and errorful learning: the effect on the discrete throwing task
}

\author{
Abdul Muiz Nor Azmi®, Jatifah Gesikin®, and Brynoth Alarick Larry®
}

Faculty of Psychology and Education, Universiti Malaysia Sabah, 88400, Sabah, Malaysia.

\begin{abstract}
The current study aimed to investigate the effect of errorless learning (ELL) and errorful learning (EFL) on a discrete aiming task (dart-throwing). Thirty university students (male, $\mathrm{n}=15$; female, $\mathrm{n}=$ $15)$ with age of $22 \pm 2.0$ years old $(\mathrm{M}=21.2 ; \mathrm{SD}=1.08)$ without any prior experience in dart-throwing participated in this study. After the pre-experimental evaluation, participants were randomly assigned into two groups (ELL and EFL group) by matched-pair design. There were three phases in the study, which were the acquisition, retention, and transfer phases. Both groups did not receive any instructions regarding the dart-throw. The results of variable error showed that there was a significant reduction in the ELL group over trials seemed to indicate that participants in ELL can utilize the learning to become more consistent in dart-throwing performance compared to the EFL group. The results in retention and transfer phases showed that the ELL group performed with significantly less error than the EFL group. The ELL group practiced with errorless learning performed with increased consistency during the retention phase compared to participants who practiced with errorful learning. The study concluded that implicit learning in errorless learning has been beneficial for the individual to get better dartthrowing performance as it can reduce the errors and lower cognitive demand when performing the task.
\end{abstract}

Keywords. Cognitive demand, dart-throw, errorless learning, errorful learning, motor learning.

\section{Introduction}

Implicit learning is unintentional learning in which is related to the passive accumulation of task knowledge that is a nonconscious process and difficult to verbalize (Masters \& Maxwell, 2004; Masters et al., 2013). In other words, individuals are not aware of the mechanisms underlying such performance improvement which allows performance to remain stable under pressure (Lam et al., 2010; Masters et al., 2008). Beek (2000) stated that implicit learning is necessary to adapt the skills when completing a critical part in motor learning. Previous studies showed that implicit learners produced a better improvement in performance through practice where the learners required a certain little amount of working memory or attention mechanisms than explicit learners, which were less prone to disturbance from secondary tasks (Liao \& Masters, 2001).

Explicit motor learning can be defined as learning produced by the process relies on working memory with the verbal knowledge of the information about movement performance (Masters \& Maxwell, 2004). Dornyei (2009) stated that explicit learning refers to the learner's consciousness or awareness during mastering some skills and materials or solving problems. Explicit learning also referred to a learning situation in which the individuals use problem- 
solving strategies to enhance their performances by generating hypotheses to gain knowledge (Berry et al., 1993; Liao \& Masters, 2001). In contrast to implicit learners, explicit learners are consciously aware of the information that has been processed in which they might share the information regarding the performance with others (Seger, 1994).

Errorless learning (ELL) is a learning strategy in which errors are reduced as much as possible or fewer errors have been made in learning new skills (Scheper, de Bruijn, Bertens, Kessels \& Brazil, 2019). ELL is based on implicit learning, which often has been used to improve learning abilities and also helps the amnesic patient from giving wrong answers (Kessels \& Haan, 2003). For instance, individuals learn something new by doing with their own rather than being corrected or shown by someone. The most important part of the ELL method is vanishing cues, where the amount of information that provides the correct technique is reduced over time. Comparatively, errorful learning (EFL) is a situation where an individual makes many errors and encourages them to guess the correct techniques when solving the problems (Metzler-BaddEley \& Snowden, 2005).

The previous studies show that individuals who get memory-impaired due to brain injury can get an advantage from errorless learning (Baddeley \& Wilson, 1994; Hammer et al., 2013; Squires et al., 1997). Baddeley \& Wilson (1994) proposed that the errorless learning method could reduce the learning errors where it would use less working memory participation for individuals when performing a task. The study from Maxwell et al. (2001) has shown that the errorless learners in golf putting performance were uninfluenced by the enforcement of a secondary task load, whereas the performance of errorful learners declined. The reduction of errors during the learning tasks would give benefit for individuals to learn the task implicitly with unselective learning processes where performance unaffected by the distraction (Maxwell et al., 2001).

Currently, there is a little study focusing on the effect of implicit learning and explicit learning on sports performance, especially in dart-throwing. Dart-throwing is considered as a closed skill in which it is performed in a stable environment. In this case, visual information acts as a provider of feedback that continuously remains and rectifies the skills as it is executed (Vickers et al., 2000). However, the question is how an implicit or explicit learning process can improve the performance of dart-throwing among adolescents. Therefore, this study aimed to investigate the effect of errorless learning (ELL) and erroful learning (EFL) in a dartthrowing task. We hypothesized that errorless and errorful learning could affect the result in a dart-throwing performance in which the ELL would be beneficial for learners to get better performance.

\section{Methods}

\section{Participants}

A total of thirty undergraduate students from the Universiti Malaysia Sabah (male, $\mathrm{n}=15$; female, $\mathrm{n}=15)$ with age of $22 \pm 2$ years old $(\mathrm{M}=21.2$; $\mathrm{SD}=1.08$ ) volunteered to participate in this study. Based on statistical power analysis, 30 sample sizes were adequate to obtain the stable result in the study where the effect size was set at 0.95 with an alpha value, 0.05 , and the power, 0.7 . The participants were randomly assigned using a matched pair design to two groups, Errorless group (ELL) $(\mathrm{n}=15)$ and Errorful group (EFL) (n $=15$ ) (see pre-experiment evaluation). All participants have no prior experience in a dartthrowing. All participants were using the right hand to perform a dart-throw. Participants have been provided informed consent before committing the experiment. The Ethics Committee of Human Research in Universiti Malaysia Sabah approved the study. 


\section{Apparatus and Tasks}

The equipment used in this study was 6 darts, a modified dartboard with a diameter of $451 \mathrm{~mm}$, and measuring tape. Participants need to throw the dart into a target (a modified dartboard which consists of 3 concentric circles). The innermost circle was allocated 3 points and the outermost circle has a score of 1 . If participants failed to hit the target (modified dartboard) and also outside the range of the circle, zero points were awarded. No throwing instructions were given to both groups since it may affect the verbal knowledge among participants.

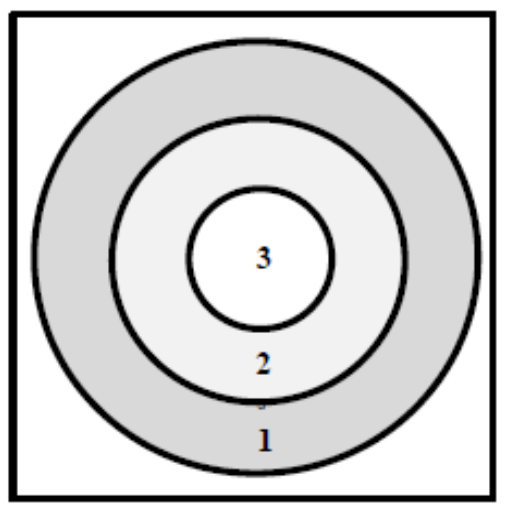

Figure 1. Modified dartboard.

\section{Experimental Design}

The current study was conducted on two consecutive days. On day 1 , before the participants went to the acquisition phase, they needed to go through the pre-experiment evaluation. This evaluation aimed to divide the participant into two groups (ELL and EFL group). On day 2, the participants were tested under 2 phases, which were retention and transfer phases.

Pre-experiment evaluation: A pre-experiment evaluation was conducted to divide participants into two groups randomly by using a matched pair design. The dartboard was set up on the wall with the distance between wall and participants is $2.2 \mathrm{~m}$ while the height from the middle of the dartboard to the floor is $1.73 \mathrm{~m}$ (height for every phase are the same). 10 trials were given to each participant to throw the darts. The score for each trial was recorded and analyzed. Participants were ranked from the highest to the lowest based on their performance. Then, they have been divided by pairs into two groups randomly (ELL and EFL group).

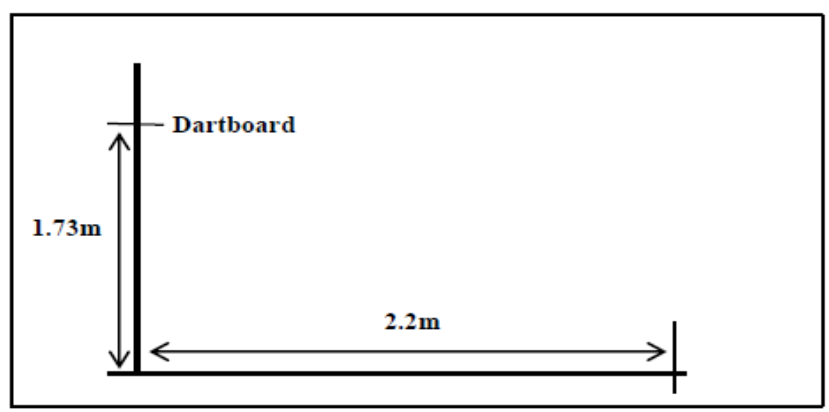

Figure 2. Simple diagram of pre-experiment evaluation test.

Acquisition Phase: The acquisition phase consists of 2 sessions with 5 blocks of 20 trials in each session and 90 seconds rest between blocks. The starting distance between participant and the target for the Errorless group (ELL) was $1.2 \mathrm{~m}$ for the 1st block with an increment of $0.2 \mathrm{~m}$ for the following blocks, which were 1.4, 1.6, 1.8, and $2.0 \mathrm{~m}$. For the Errorful group (EFL), the starting distances between the participant and the target was $2.8 \mathrm{~m}$ with a decrement of $0.2 \mathrm{~m}$ for the next following blocks $-2.6,2.4,2.2$, and $2.0 \mathrm{~m}$. The differences in throwing distances between groups were determined by the crossover design, which aims to compare the effect of different treatments on participants (Zheng, 2013). The ELL group started with a short distance $(1.4 \mathrm{~m})$, while the EFL group started at a far distance $(2.8 \mathrm{~m})$. The scores for both groups were recorded.

Retention Test: The retention test was carried out at least one day (24 hours) after the acquisition 
phase. This phase consists of 1 block of 20 trials. Both groups used the same throwing distance in which the distance between participants and the targets was $2.0 \mathrm{~m}$. The scores for both groups were recorded.

Transfer Test: The transfer test was carried out 15 minutes after the retention phase finished. Transfer test consists of 1 block of 20 trials for both groups; the Errorless group and Errorful group with the standard distance of dart throwing was $2.37 \mathrm{~m}$. The scores for both groups were recorded.

\section{Statistical Analysis}

In the present study, the descriptive analysis was reported as mean \pm standard deviation (SD). The dependent variable in this study was the variable error. The variable error (VE) was used to measure the consistency, calculated as the within-subject variability over a series of trials between both groups for each phase. For the acquisition block trials, analysis of variance (ANOVA) with repeated measures was used to determine any changes in the dependent variable between the ELL or EFL group. The post hoc test with Bonferroni correction was used to determine the difference between the time points for both groups. For the retention and transfer phases, an independent sample t-test was used to determine the difference of the dependent variable between the ELL and EFL group. The effect size (ES) was also reported by using Rhea's (2004) suggested ES values, $<.025$ is trivial, 0.250.50 represents a small, $0.50-1.00$ represents a medium, $>1.0$ is considered large. The significant level was set as $<0.05$ using a statistical software package (SPSS Inc., Chicago, IL, USA, 22.0).

\section{Results}

Table 1 shows the means (SD) for performance consistency (variable error - VE) of the participants for both groups (ELL and EFL) during the acquisition block trials, retention, and transfer phases. For the acquisition phase in block 1 and block 2, there were statistically significant differences between the ELL and EFL group, $\mathrm{F}(1,28)=4.277$ and 8.331, $\mathrm{p}<.05$. However, the result for trials in block 3 showed that there was no statistically significant difference between both groups, $\mathrm{F}(1,28)=3.671$, $\mathrm{p}>.05$. The results for trials in blocks 4 and 5 revealed that there were statistically significant differences between the ELL and EFL groups, F $(1,28)=6.508$ and 8.192, $p<.05$. Based on the results, we can see that the ELL group performed with significantly less error than the EFL group across the acquisition block trials. For the retention and transfer phases, the results revealed that there was a significant difference between group ELL and EFL in retention and transfer phases, $\mathrm{t}(28)=10.202, \mathrm{p}<.05, \mathrm{t}(28)=$ 8.056, $\mathrm{p}<.05$, respectively. Based on Figure 1, we can see that when using a new distance in the transfer phase, both groups had significantly greater errors compared to the acquisition and retention phase. However, the ELL group still performed a greater consistency when recorded a lower VE compared to the EFL group.

\section{Discussion}

This study aimed to examine the effect of errorless learning and errorful learning in dartthrowing and also comparing the dart-throwing performance between the ELL group and the EFL group. We hypothesized that errorless learning would be more beneficial to learning than errorful learning, which can reduce the errors consistently. Participants' performances in dart-throwing were assessed during the acquisition block trials, retention, and transfer phases. Based on the results in Table 1 and Figure 3, it showed that the ELL group recorded the lowest dependent variable in all three phases compared to the EFL group. It means that the ELL group has better consistency in dartthrowing performance than the EFL group in all 


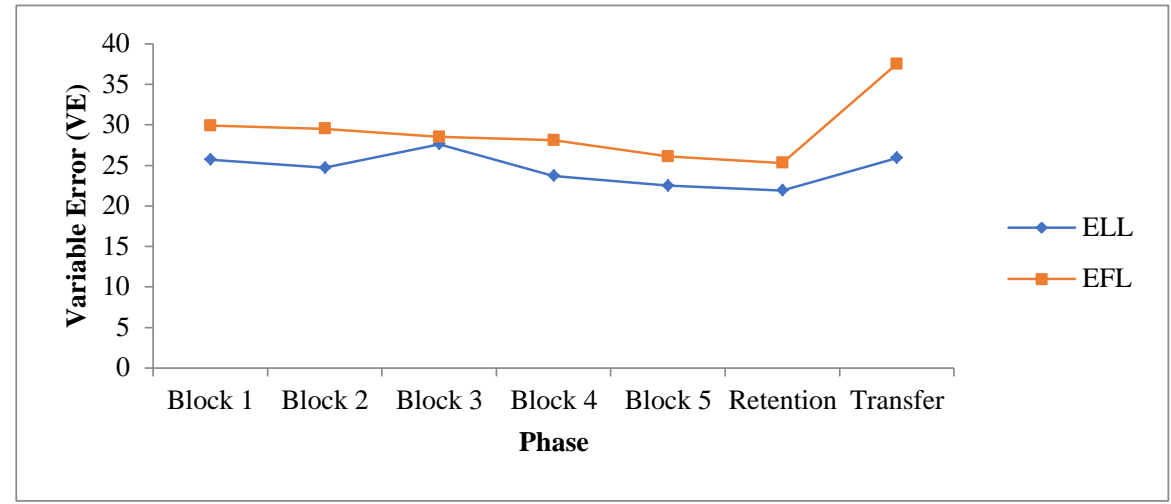

Figure 3. Block means for variable error (VE) during acquisition, retention, and transfer phases for ELL and EFL groups.

\begin{tabular}{|c|c|c|c|c|}
\hline Variables & ELL & EFL & $p$ & ES \\
\hline Block 1 & 25.7 (1.6) & 29.9 (1.8) & .002 & .234 \\
\hline Block 2 & $24.7(2.5)$ & $29.5(2.1)$ & .001 & .088 \\
\hline Block 3 & $27.6(2.1)$ & 28.5 (1.8) & .113 & .245 \\
\hline Block 4 & 23.7 (1.7) & 28.1 (1.6) & .012 & .711 \\
\hline Block 5 & $22.5(1.8)$ & $26.1(2.3)$ & .009 & .451 \\
\hline Retention & 21.9 (1.8) & $25.3(1.8)$ & .002 & .422 \\
\hline Transfer & $25.0(1.2)$ & $37.5(2.0)$ & .007 & .696 \\
\hline
\end{tabular}

three phases. Based on the results in Table 1, both groups recorded the lowest VE in the retention phase. However, the dependent variable was not maintained over the trials in the transfer phase as the participants recorded a significantly greater dependent variable but still, the ELL group was more consistent when recorded the lowest VE compared to the EFL group.

It showed that errorless learning is more advantageous than errorful learning, which can enhance skill learning and reduce the usage of memory. Lam et al. (2010) suggested that the attentional capacity of individuals remained constant in which there were no changes in attention requirements as the throwing distance increased. The condition in ELL has facilitated the participants to reduce the errors while performing the task where participants have begun the practice in the acquisition phase with the nearest target. The automatic processing in errorless learning can be due to the lack of working memory accumulation (fewer errors) (Poolton et al., 2005). The reduction of the errors early in the acquisition phase has made attention resources to be used more effectively by participants in ELL during performing the skill and when the task demands changed.

In contrast, participants in the EFL group have the attention capacity constraints while performing the task which made it difficult to aim the target as the throwing distance was far in the beginning phase (acquisition phase). They are unable to distinguish between tasks and follow effortful processes. The vigilant processing in EFL is due to the working memory 
accumulation in terms of errorful (Beilock \& Carr, 2001). Errorful learning is promoting the explicit processes in which rely on working memory capacity and manipulation of information. At the same time, errorless learning is promoting implicit learning in which typically unavailable for conscious inspection and without verbal knowledge of the skill (Masters \& Maxwell, 2004). The current findings are consistent with the previous study where they found that errorless learning can be made with mentally and physical effortlessness; yet achieve higher levels of performance (Abdoli et al., 2013; Lam et al., 2010).

Moreover, errorless learning also leads to more automated movement control which can reduce the intentional areas and consist of a clear mind throughout the activity. The presence of errorless learning can help to minimize the performance error that can lead to an increase in skills proficiency (Lam et al., 2010). Besides, errorless learning is suitable to use to teach beginners like children because the learning does not require greater attentional capacities. It can help children to process the information well as their information processing tends to be slower (Ferguson \& Bowey, 2005). It could lead children to produce better performance with fewer errors made when performing the tasks (Abdoli et al., 2013; Masters, 1992; Poolton et al., 2005). Hence, it can be seen that the performance can be improved or skill can be acquired easier if there is less constraint faced by an individual during the process of motor learning. For instance, children can start learning the way of passing in basketball by using a smaller and lighter ball in a shorter distance during practice.

A further question of interest is whether there are different ways or protocols of errorless learning on motor control that can be studied. To obtain more reliable results on the effect of errorless learning on the discrete aiming task, we suggest that the participants or the subjects are from teenager or children categories as a reason most of them are not skilled enough on such correlated skills. We would hope that other researchers would investigate the effect of errorless learning in other sports.

\section{Acknowledgement}

The authors would like to thank the participants from the Universiti Malaysia Sabah who volunteered to participate in the study.

\section{Funding}

There was no funding for this study.

\section{Conflict of Interest}

The authors have no conflicts of interest to declare.

\section{References}

Abdoli, B., Farsi, A., \& Barani, F.H. (2013). The effects of errorless and errorful practices on learning effort. International Journal of Sport Studies, 3(4), 472-479.

Baddeley, A., \& Wilson, B.A. (1994). When implicit learning fails: Amnesia and the problem of error elimination. Neuropsychologia, 32(1), 53-68.

Beek, P.J. (2000). Toward a theory of implicit learning in the perceptual-motor domain. In Scientific Sport Psychology Workshop, Nov, 1997, Amsterdam, Netherlands: Edizioni Luigi Pozzi.

Beilock, S.L, \& Carr, T.H. (2001). On the fragility of skilled performance: what governs choking under pressure? J Exp Psychol Gen, 130, 701-725.

Berry, D. C., Barry, D. P., Berry, D., \& Dienes, Z. (1993). Implicit learning: Theoretical and empirical issues. Psychology Press.

Dornyei, Z. (2009). The psychology of second language acquisition. Oxford: Oxford University Press.

Ferguson, A.N., \& Bowey, J.A. (2005). Global processing speed as a mediator of developmental changes in children's auditory memory span. J Exp Child Psychol, 91(2), 89-112.

Hammer, A., Heldmann, M., \& Münte, T.F. (2013). Errorless and errorful learning of face-name 
associations: an electrophysiological study. Bio Psychol, 92(2), 169-178.

Kessels, R. P., \& Haan, E. H. (2003). Implicit learning in memory rehabilitation: A meta-analysis on errorless learning and vanishing cues methods. J Clin Exp Neuropsychol, 25(6), 805-814.

Lam, W.K., Maxwell, J.P., \& Masters, R.S.W. (2010). Probing the allocation of attention in implicit (motor) learning. J Sports Sci, 28(14), 1543-1554.

Liao, C.M., \& Masters, R.S. (2001). Analogy learning: a means to implicit motor learning. J Sports Sci, 19(5), 307-319.

Masters, R.S.W., \& Maxwell, J.P. (2004). Implicit motor learning, reinvestment and movement disruption: What you don't know won't hurt you? In A.M. Williams \& N.J. Hodges (Eds.), Skill acquisition in sport: Research, theory and practice. London: Routledge.

Masters, R.S.W., van der Kamp, J., \& Capio, C. (2013). Implicit motor learning by children. Conditions of children's talent development in sport. West Virginia: Fitness Information Technology, 21-40.

Masters, R.S. (1992). Knowledge, knerves and knowhow: The role of explicit versus implicit knowledge in the breakdown of a complex motor skill under pressure. Br J Psychol, 83(3), 343-358.

Masters, R.S.W., Poolton, J.M., Maxwell, J.P., \& Raab, M. (2008). Implicit motor learning and complex decision making in time-constrained environments. J Mot Behav, 40(1), 71-79.

Maxwell, J.P., Masters, R.S.W., Kerr, E., \& Weedon, E. (2001). The implicit benefit of learning without errors. The Q J Exp Psychol Section A,54(4), 10491068.

Maxwell, J.P. (2001). The implicit benefit of learning without errors. Q J Exp Psychol, 54A, 1049-1068.

Metzler-BaddEley, C., \& Snowden, J.S. (2005). Brief report: errorless versus errorful learning as a memory rehabilitation approach in Alzheimer's disease. J Clin Exp Neuropsychol, 27(8), 1070-1079.

Poolton, J.M., Masters, R.S.W., \& Maxwell, J.P. (2005). The relationship between initial errorless learning conditions and subsequent performance. Hum Mov Sci, 24(3), 362-378.

Rhea, M. R. (2004). Determining the magnitude of treatment effects in strength training research through the use of the effect size. J Strength Cond Res, 18, 918-920.

Scheper, I., de Bruijn, E.R., Bertens, D., Kessels, R.P., \& Brazil, I.A. (2019). The impact of error frequency on errorless and errorful learning of object locations using a novel paradigm. Memory, 27(10), 1371-1380.

Seger, C.A. (1994). Implicit learning. Psychological Bulletin, 115(2), 163.

Squires, E. J., Hunkin, N. M., \& Parkin, A. J. (1997). Errorless learning of novel associations in amnesia. Neuropsychologia, 35(8), 1103-1111.

Vickers, J. N., Rodrigues, S. T., \& Edworthy, G. (2000). Quiet eye and accuracy in the dart throw. International Journal of Sports Vision, 6(1), 3036.

Zheng, W. (2013). Universally optimal crossover designs under subject dropout. Ann Stat, 41(1), 6390. 